\title{
Case report: two novel VPS13B mutations in a Chinese family with Cohen syndrome and hyperlinear palms
}

\author{
Sha Zhao ${ }^{1,2 \dagger}$, Zhenqing Luo ${ }^{1 \dagger}$, Zhenghui Xiao ${ }^{1}$, Liping Li ${ }^{1}$, Rui Zhao ${ }^{1}$, Yongjia Yang ${ }^{1 *}$ (D) and Yan Zhong ${ }^{2^{*}}$
}

\begin{abstract}
Background: Cohen syndrome (CS) is an uncommon developmental disease with evident clinical heterogeneity. VPS13B is the only gene responsible for CS. Only few sporadic cases of CS have been reported in China.

Case presentation: A Chinese family with two offspring-patients affected by developmental delay and intellectual disability was investigated in this study. Exome sequencing was performed, and compound heterozygous mutations in VPS13B were segregated for family members with autosomal recessive disorder. Splicing mutation c.3666 + 1G > T (exon 24) and nonsense mutation c. 9844 A > T:p.K3282X (exon 54) were novel. We revisited the family and learned that both patients are affected by microcephaly, developmental delay, neutropenia, and myopia and have a friendly disposition, all of which are consistent with CS phenotypes. We also found that both patients have hyperlinear palms, which their parents do not have. VPS13B mutations reported among the Chinese population were reviewed accordingly.
\end{abstract}

Conclusions: This study presents two novel VPS13B mutations in CS. The identification of hyperlinear palms in a family affected by CS expands the phenotype spectrum of CS.

Keywords: Cohen syndrome, Hyperlinear palm, VPS13B gene, Mutation, Chinese

\section{Background}

Cohen syndrome (CS) (MIM\# 216550), a rare disorder, was initially described by M. Michael Cohen, Jr., and his colleagues in 1973 [1]. CS is a clinically heterogeneous disorder mainly characterized by developmental delay, intellectual disability, microcephaly, and hypotonia with highly variable clinical findings on myopia, retinal dystrophy, joint hypermobility, neutropenia, overly friendly behavior, truncal obesity, slender fingers, and facial appearance consisting of thick hair, thick eyebrows, long eyelashes, down-slanting eyes, short philtrum, and prominent upper incisors [2].

The clinical heterogeneity and several phenotypes of CS are unobservable before 10 years old; thus, this rare

\footnotetext{
* Correspondence: yongjia727@aliyun.com; zhongyan@163.com

+'Sha Zhao and Zhenqing Luo contributed equally to this work.

'The Laboratory of Genetics and Metabolism, Hunan Children's Research Institute (HCRI), Hunan Children's Hospital, University of South China, Changsha 410007, China

${ }^{2}$ Department of Child Healthcare, Hunan Children's Hospital, University of South China, Changsha 410007, Hunan, China
}

disease is difficult to diagnose in clinical practice. Nevertheless, CS can now be diagnosed through VPS13B mutation screening with the cloning of the disease-causing gene VPS13B [3-5]. VPS13B is the only gene that causes CS. Several VPS13B mutations have been recently reported in families with CS in Tunisia and Pakistan $[6,7]$.

Human VPS13B (NM_017890), which is located on $8 \mathrm{q} 22.2$, consists of 62 exons that encode a 4022-amino acid transmembrane protein of the Golgi apparatus functioning in vesicle-mediated transport and sorting of proteins within the cell [3]. Approximately 200 VPS13B mutations (http://www.hgmd.cf.ac.uk/) have been reported in nearly 1000 CS patients worldwide (http://www. cohensyndrome.org/). Founder mutations have been described in several areas [2]. Only a few VPS13B mutations have been reported sporadically in patients with CS (two definitive CS and three probable CS) in the Chinese population [8-10]. In the present study, exome sequencing identified two novel VPS13B mutations in a Chinese family with two offspring-patients affected by CS and

(c) The Author(s). 2019 Open Access This article is distributed under the terms of the Creative Commons Attribution 4.0 International License (http://creativecommons.org/licenses/by/4.0/), which permits unrestricted use, distribution, and reproduction in any medium, provided you give appropriate credit to the original author(s) and the source, provide a link to the Creative Commons license, and indicate if changes were made. The Creative Commons Public Domain Dedication waiver (http://creativecommons.org/publicdomain/zero/1.0/) applies to the data made available in this article, unless otherwise stated. 
hyperlinear palms. The presence of hyperlinear palms can likely expand the phenotypic spectrum of CS.

\section{Case presentation}

The study protocol was approved by the Academic Committee of Hunan Children's Hospital (Approval No. HCHLL58, Changsha City, Hunan Province, China). The four family members (Han Chinese ethnicity), which included two parents and two children, provided written informed consent to participate in this study. Parental permission for publishing patients' photos was obtained. The genomic DNAs of all four family members were isolated using standard methods.

\section{Patient 1}

The propositus was the first child born to the healthy nonconsanguineous parents (father's age: 27; mother's age: 22) after a spontaneous uncomplicated delivery with Apgar scores of 9 and 10. The patient's birth weight was $2900 \mathrm{~g}$ (25th-50th centile), and his length was $48.5 \mathrm{~cm}$ (25th-50th centile). He was evaluated when he was 7.5 years old with a height of $111.4 \mathrm{~cm}(-3.02 \mathrm{SD})$, weight of $23.5 \mathrm{~kg}$ (25th-50th centile), and occipitofrontal circumference (OFC) of $45.2 \mathrm{~cm}$ (normal reference: 52.6 $\mathrm{cm})$. The patient has been walking alone since he was two years old, but he cannot complete a sentence (i.e., more than two words). The patient exhibits hyperactivity, disobedience, severe mental retardation, generalized joint hyperextensibility, hypotonia, slender fingers, hyperlinear palm (Fig. 1a), and special facial features, including prominent upper central incisors, thick hair, thick eyebrows, micrognathia, prominent upper lip, and prominent root of the nose. Neutropenia (neutrophil count: $0.58 \times 109 / \mathrm{L}$, normal range: $2.00-7.00 \times 109 / \mathrm{L}$ ) was observed when the patient was 8.2 years old and occurred after a cold. Truncal obesity was unobservable.

\section{Patient 2}

The propositus' younger sister was evaluated when she was 5.8 years old. Her height was $97.5 \mathrm{~cm}(-3.72 \mathrm{SD})$, her weight was normal, and her OFC was $43.5 \mathrm{~cm}$ (normal reference: $51.5 \mathrm{~cm}$ ). She has been walking alone since she was 2.5 years old, but she cannot complete a sentence. Similar to her older brother, she exhibits severe mental retardation, generalized joint hyperextensibility, and hypotonia. Her special facial features include prominent upper central incisors, thick hair, thick eyebrows, long and thick eyelashes, bilateral ptosis, bilateral epicanthus inversus, and bilateral strabismus. Neutropenia and truncal obesity were unobservable. The patient has hyperlinear palms (Fig. 1b).

\section{Genetic testing}

CS in the Chinese population has rarely been reported. Neutropenia was unobservable in the selected family before this experiment. GTG banding was initially performed, and the karyotypes of the four family members were normal. Then, a copy number variation (CNV) test for the propositus (M2650, Fig. 2a) was performed with the Illumina Human OmniZhongHua-8 BeadChip array (Illumina, San Diego, CA, USA). No pathogenic CNV was detected, and the family likely suffered from a monogenetic disease. Exome sequencing of the four members was performed according to a previously described pipeline [11]. No consanguineous marriage in the family was reported. The severe phenotypes of both offspring (but none of the parents) exhibited abnormalities. We focused on genes/variants that met the following criteria: (1) coding region variants with MAF $<0.001$ (All_gnomAD, ExAc, 1000 genomes, and ESP6500); (2) damaging variants consisting of loss-of-function (LOF) and damaging missense variants (VarCards); (3) recessive inheritance gene consisting of compound heterozygous or homozygous variants; and (4) the gene function related to the phenotypes. Only one gene (compound heterozygous variant), that is, $V P S 13 B$ (data not shown), was selected. Sanger sequencing was performed (the primer and PCR condition are provided in Additional file 1: Table S1). The two LOF variants of VPS13B (chr8:100479863G > T, NM_017890c. $3666+1 \mathrm{G}>\mathrm{T}$ and chr8:100847793A $>$ T, c.9844A $>\mathrm{T}$ :

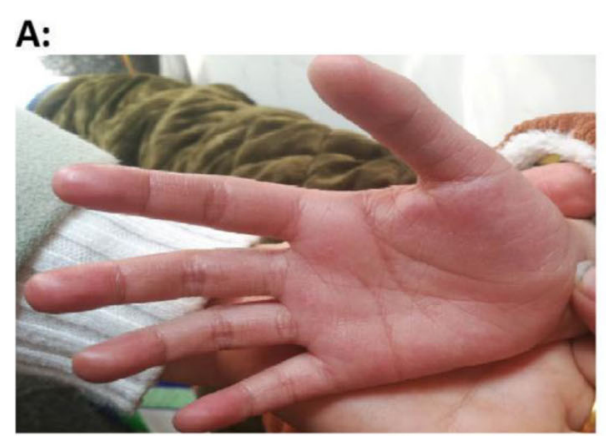

B:

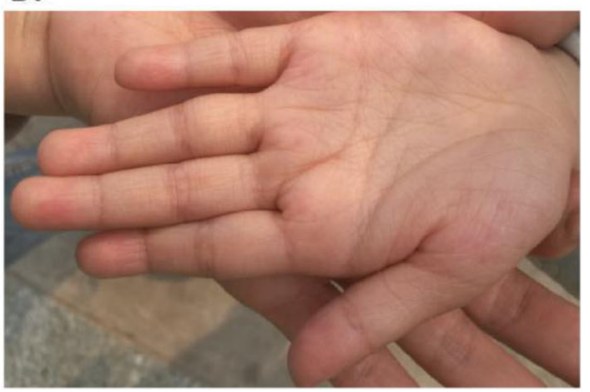

Fig. 1 Patients with CS in a Chinese family. a Left hand palm of female patient M2651 and $\mathbf{b}$ Right hand palm of male patient M2650 


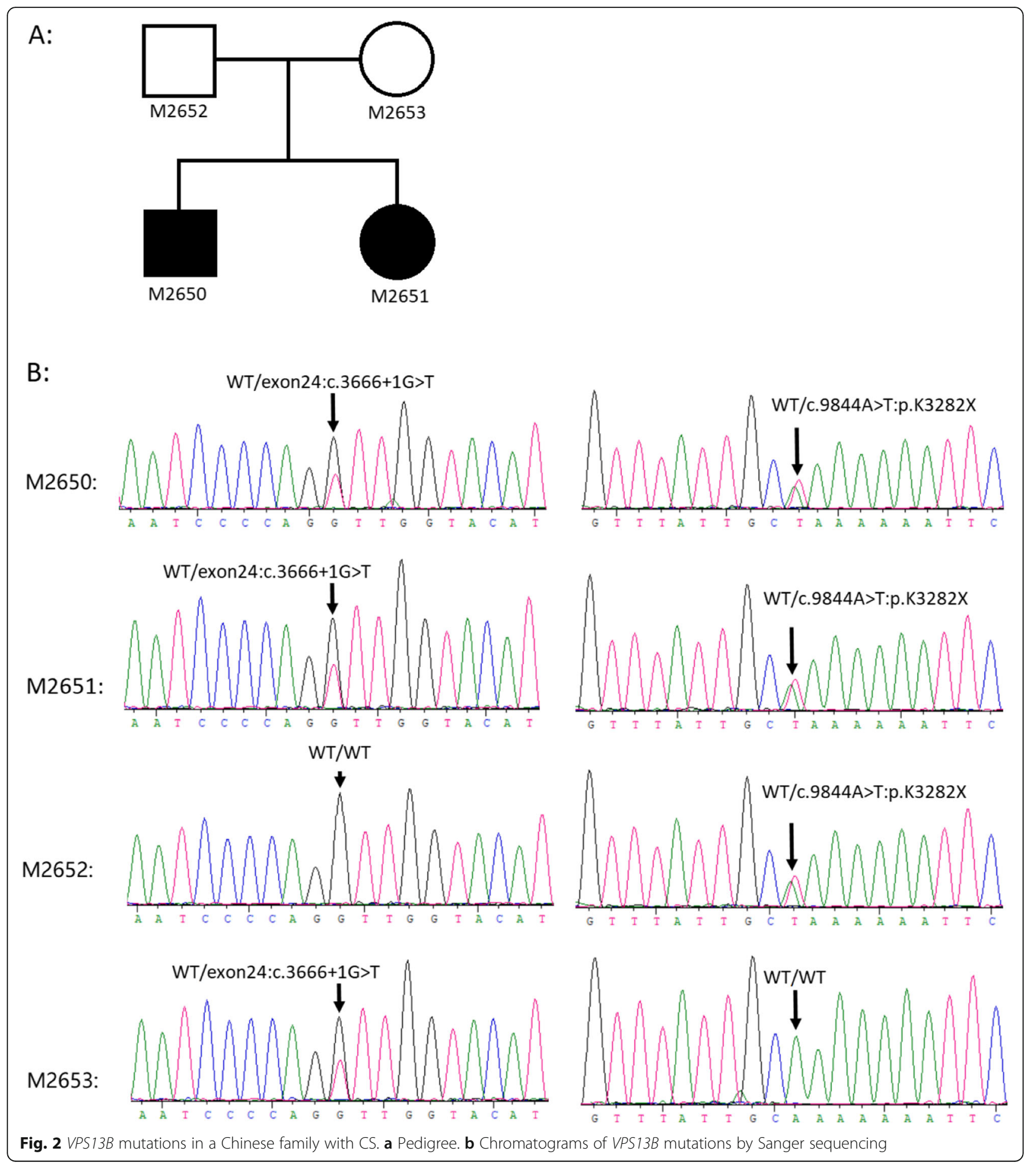

p.K3282X) were confirmed, and the VPS13B variants were co-segregated with the disorder in autosomal recessive mode in the family (Fig. 2b).

\section{Discussion and conclusions}

A family with two offspring-patients affected by severe mental retardation and speech delay came to our clinic for genetic counselling. The detection of compound heterozygous mutations in VPS13B via exome sequencing directed us to the diagnosis of CS in the family. CS is a clinical heterogeneous disorder whose occurrence is high among patients with different ethnic backgrounds [12]. For example, patients who belong to Finnish and Greek cohorts present much richer skeletal phenotypes featured by 


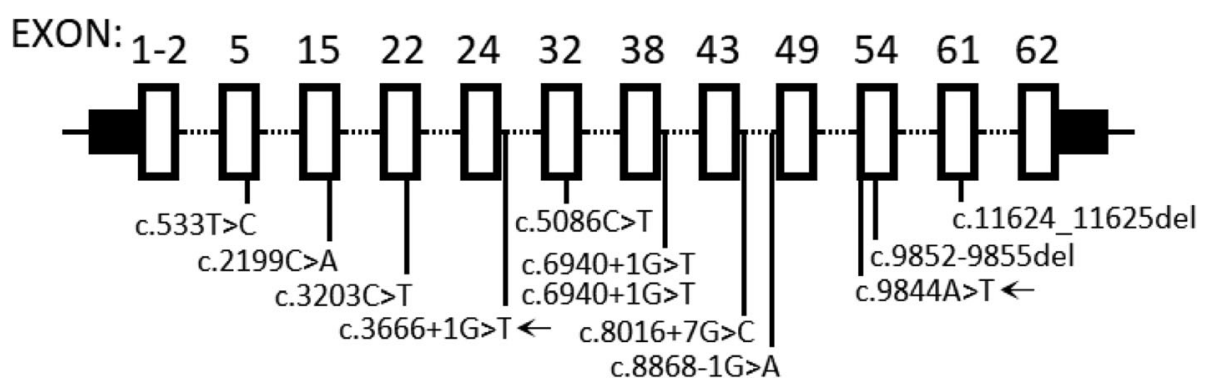

Fig. 3 Schematic presentation of VPS13B mutations in the Chinese population. The arrow represents the mutations identified in the present study

slender extremities and/or tapered fingers, joint hypermobility, and sandal gap compared with patients who belong to the same cohort $[12,13]$.

The two patients with CS in this study have slim or slender fingers. Hyperlinear palms were also observed in the two patients but not in their parents and other family members (Fig. 1). Hyperlinear palms or palms with extra skin creases signify a hand anomaly ("https://www.rightdiagnosis.com/symptom/hyperlinear-palms-extra-skincreases-in-the-palms.htm"). Hyperlinear palms have been reported among FLG-mutated patients [14] with atopic dermatitis or ichthyosis vulgaris [15]. We tried contacting VPS13B-mutated Chinese patients [8-10] to see if they have hyperlinear palms as a means of verifying if hyperlinear palms are a component phenotype of CS. However, we have not received any response from these patients. Therefore, additional supporting data are required to determine if hyperlinear palms are a CS phenotype.

CS was initially described in 1973 [1], and VPS13B mutation in CS was reported in 2003 [3]. However, only two definitive CS $[8,9]$ and three probable CS patients have been reported in the Chinese population; the first patient with CS was diagnosed by next-generation sequencing in 2016 [8]. In the present study, we reviewed all VPS13B mutations reported in the Chinese population (Fig. 3). Only one $V P S 13 B$ mutation $(c .6940+1 \mathrm{G}>\mathrm{T})$ was reported twice, and the others in the VPS13B gene were evenly distributed. Further studies involving Chinese patients with CS are needed to determine if founder mutation of VPS13B occurs among Chinese families.

This study identified two novel VPS13B mutations in a Chinese family with two members affected by CS. This work is the first to report a complete set of CS data involving a Chinese family. The presence of hyperlinear palms (identified in this study) is likely a novel phenotype of CS and may provide new clues for CS diagnosis.

\section{Supplementary information}

Supplementary information accompanies this paper at https://doi.org/10. 1186/s12881-019-0920-x.

Additional file 1: Table S1. Primers and PCR conditions in this study.

\section{Abbreviations \\ CNV: Copy number variation; CS: Cohen syndrome; LOF: Loss of function; OFC: Occipitofrontal circumference; PCR: Polymerase chain reaction}

Acknowledgements

Gratitude is extended to the subject family for their cooperation and participation.

\section{Authors' contributions}

YJY, RZ, LPL and YZ designed the study, monitored the family investigation, collected clinic data, conducted experiments, and revised the manuscript. YJY, ZQL, ZHX, LPL and SZ contributed to exome sequencing, PCR experiments, Sanger sequencing, and drafting the paper. SZ and YJY analyzed the clinical data. YJY, LLP, and SZ contributed to sample collection and genetic counselling. All authors read and approved the final manuscript.

\section{Funding}

This work was supported by the National Natural Science Foundation of China (31501017, to Yongjia Yang), Hunan Health Commission Research Fund (B2019019, to Yongjia Yang), and Key Laboratory Fund of Hunan Province (2018tP1028, to Zhenghui Xiao). The funding body had no influence in the in the design of the study and collection, analysis, and interpretation of data and in writing the manuscript.

\section{Availability of data and materials}

All data generated or analyzed during this study are included in this published article.

\section{Ethics approval and consent to participate}

The study protocol was approved by the Academic Committee of Hunan Children's Hospital (Approval number: HCHLL58, Changsha City, Hunan Province, China). The four family members provided their written informed consent to participate in this study.

\section{Consent for publication}

Written informed consent for publication was obtained from the participants or their guardians.

\section{Competing interests}

The authors declare that they have no competing interests.

Received: 25 June 2019 Accepted: 11 November 2019

Published online: 21 November 2019

\section{References}

1. Cohen MM Jr, Hall BD, Smith DW, et al. New syndrome with hypotonia, obesity, mental deficiency and facial, oral, ocular and limb anomalies. J Pediatr. 1973;83:280-4.

2. Rodrigues JM, Fernandes HD, Caruthers C, et al. Cohen Syndrome: Review of the Literature. Cureus. 2018;10(9):e3330.

3. Kolehmainen J, Black GC, Saarinen A, et al. Cohen syndrome is caused by mutations in a novel gene, $\mathrm{COH}$, encoding a transmembrane protein with a presumed role in vesicle-mediated sorting and intracellular protein transport. Am J Hum Genet. 2003;72(6):1359-69. 
4. Kolehmainen J, Wilkinson R, Lehesjoki AE, et al. Delineation of Cohen syndrome following a large-scale genotype-phenotype screen. Am J Hum Genet. 2004;75(1):122-7.

5. Hennies HC, Rauch A, Seifert W, Schumi C, et al. Allelic heterogeneity in the $\mathrm{COH} 1$ gene explains clinical variability in Cohen syndrome. Am J Hum Genet. 2004;75(1):138-45

6. Rejeb I, Jilani H, Elaribi Y, Hizem S, Hila L, Zillahrdt JL, Chelly J, Benjemaa L. First case report of Cohen syndrome in the Tunisian population caused by VPS13B mutations. BMC Med Genet. 2017;18(1):134.

7. Rafiq MA, Leblond CS, Saqib MA, Vincent AK, Ambalavanan A, Khan FS, Ayaz M, Shaheen N, Spiegelman D, Ali G, Amin-ud-Din M, Laurent S, Mahmood H, Christian M, Ali N, Fennell A, Nanjiani Z, Egger G, Caron C, Waqas A, Ayub M, Rasheed S, Forgeot d'Arc B, Johnson A, So J, Brohi MQ, Mottron L, Ansar M, Vincent JB, Xiong L. Novel VPS13B Mutations in Three Large Pakistani Cohen Syndrome Families Suggests a Baloch Variant with Autistic-Like Features. BMC Med Genet. 2015;16:41.

8. Yin Lianhai, Cheng Mangmang, Wang Yuanxiao, et al. Case report of Cohen syndrome and literature review. Chin J Appl Clin Pediatr, 2016, Vol.31, No.19.

9. Zhang F, Shi $X Y$, Liu LY, et al. [Psychomotor retardation with neutropenia for more than one year in a toddler].[article in Chinese]. Zhongguo Dang Dai Er Ke Za Zhi. 2018;20(6):497-500.

10. Yang $C$, Hou M, Li Y, et al. Gene analysis: a rare gene disease of intellectual deficiency-Cohen syndrome. Int J Dev Neurosci. 2018;68:83-8.

11. Yang $Y$, Zheng Y, Li W, et al. SMAD6 is frequently mutated in nonsyndromic radioulnar synostosis. Genet Med. 2019. https://doi.org/10.1038/s41436-0190552-8.

12. Douzgou S, Petersen MB. Clinical variability of genetic isolates of Cohen syndrome. Clin Genet. 2011;79(6):501-6.

13. Kivitie-Kallio $S$, Norio R. Cohen syndrome: essential features, natural history, and heterogeneity. Am J Med Genet. 2001;102(2):125-35.

14. Landeck L, Visser M, Kezic S, et al. Genotype-phenotype associations in filaggrin loss-of-function mutation carriers. Contact Dermatitis. 2013;68(3): 149-55.

15. Chen $\mathrm{H}$, Common JE, Haines RL, et al. Wide spectrum of filaggrin-null mutations in atopic dermatitis highlights differences between Singaporean Chinese and European populations. Br J Dermatol. 2011;165(1):106-14.

\section{Publisher's Note}

Springer Nature remains neutral with regard to jurisdictional claims in published maps and institutional affiliations.

Ready to submit your research? Choose BMC and benefit from:

- fast, convenient online submission

- thorough peer review by experienced researchers in your field

- rapid publication on acceptance

- support for research data, including large and complex data types

- gold Open Access which fosters wider collaboration and increased citations

- maximum visibility for your research: over $100 \mathrm{M}$ website views per year

At BMC, research is always in progress.

Learn more biomedcentral.com/submissions 